\title{
Complex Structure of the Four-Dimensional Kerr Geometry: Stringy System, Kerr Theorem, and Calabi-Yau Twofold
}

\author{
Alexander Burinskii \\ Theoretical Physics Laboratory, NSI, Russian Academy of Sciences, B. Tulskaya 52, Moscow 115191, Russia \\ Correspondence should be addressed to Alexander Burinskii; bur@ibrae.ac.ru
}

Received 12 November 2012; Accepted 9 February 2013

Academic Editor: P. Bussey

Copyright ( 2013 Alexander Burinskii. This is an open access article distributed under the Creative Commons Attribution License, which permits unrestricted use, distribution, and reproduction in any medium, provided the original work is properly cited.

\begin{abstract}
The 4D Kerr geometry displays many wonderful relations with quantum world and, in particular, with superstring theory. The lightlike structure of fields near the Kerr singular ring is similar to the structure of Sen solution for a closed heterotic string. Another string, open and complex, appears in the complex representation of the Kerr geometry initiated by Newman. Combination of these strings forms a membrane source of the Kerr geometry which is parallel to the structure of M-theory. In this paper we give one more evidence of this relationship, emergence of the Calabi-Yau twofold (K3 surface) in twistorial structure of the Kerr geometry as a consequence of the Kerr theorem. Finally, we indicate that the Kerr stringy system may correspond to a complex embedding of the critical $N=2$ superstring.
\end{abstract}

\section{Introduction}

The black hole solutions of diverse dimensions represent now one of the basic objects for study in superstring theory. Recent ideas and methods in the black hole physics are based on complex analyticity and conformal field theory, which unifies the black hole physics with superstring theory and physics of elementary particles. The Kerr solution plays in this respect especial role. Being obtained as a metric of a "spinning mass" [1] with angular momentum $J=m|a|$, the Kerr solution found basic application as a metric of rotating black hole. In the four-dimensional Kerr solution, parameter $a=\mathrm{J} / \mathrm{m}$ is radius of the Kerr singular ring. For $|a|<m$, the ring is covered by horizon, but for parameters of the elementary particles $|a| \gg m$, the black hole horizons disappear, and the Kerr singular ring turns out to be naked. Following the censorship principle, it should be covered by a source. During four decades of investigations, structure of Kerr's source was specified step by step. One of the earlier models was the model of the Kerr ring as a closed string [2,3]. It has been obtained in [4] that structure of the fields around the Kerr ring is similar to the structure of the heterotic string in the solutions to low energy string theory obtained by Sen [5]. However, the Kerr string is branch line of the Kerr spacetime into two sheets [6], and this bizarre peculiarity created an alternative line of investigations of the problem of Kerr's source [7-12], which led to conclusion that the source of the Kerr-Newman (KN) solution should form a rigidly rotating membrane, or to be more precise, a highly oblate ellipsoidal bubble with a flat vacuum interior $[9,12]$.

The charged KN solution [13] has found application as a consistency with gravity classical model of spinning particle, $[2,8,9,14-16]$, which has gyromagnetic ratio $g=2$, as that of the Dirac electron $[14,15]$ (In fact, the four observable parameters of the electron: mass, spin, charge and magnetic moment indicate unambiguously that the $\mathrm{KN}$ solution is to be the electron background geometry $[17,18])$. The $\mathrm{KN}$ solution displays also other relationships with the Dirac electron [1721], as well as the relationships with twistor theory [6, 22-24], models of the soliton [10-12, 25], and with basic structures of superstring theory $[3,4,17,18,24,26-29]$.

In this paper we consider complex structure of the Kerr geometry [26-29] and reveal one new evidence of its inherent parallelism with twistor theory and superstring theory. Namely, we show the presence of the Calabi-Yau twofold (K3 surface) in complex structure of the Kerr geometry, which appears as a consequence of the Kerr theorem in the form of a quartic equation in the projective twistor space $\mathrm{CP}^{3}$. In Section 2, we describe briefly the real structure of the Kerr 
geometry and the Kerr theorem, which determines Kerr's principal null congruence (PNC) in twistor terms.

On the way to our principal result, there appear a few important intermediate structures. First of all it is the complex Kerr geometry itself, which is generated by the Appel complex shift method [30] and by the Newman complex retarded-time construction $[31,32]$. We describe them in Section 3.

In Section 4, we show that the source of the complex Kerr geometry is an open complex string. It is based on the old remarks by Ooguri and Vafa that the complex world lines (CWL) parametrized by complex time parameter $\tau=t+i \sigma$ turns into a worldsheet of a complex string, [33], and the complex Kerr string generating the complex Kerr geometry, is to be an open string with orientifold worldsheet [26-29].

Finally, we observe that the structure of the membrane source of the real Kerr geometry is parallel to formation of the membrane by the transfer from superstring theory to $\mathrm{M}$ theory: the closed Kerr string of the real Kerr geometry grows by extra worldsheet parameter from the open complex Kerr string, in analogue with the known superstring/M-theory correspondence $[34,35]$.

The parallelism of the complex Kerr geometry with the basic structures of the superstring theory and; in particular, the inherent existence of the $\mathrm{K} 3$ surface in twistorial $\mathrm{CP}^{3}$ space of the principal null congruences, allows us to suppose that the complex Kerr string represents a complex realization of the critical $N=2$ superstring theory, embedding of which into complex Kerr geometry looks admissible, leading to the point of view that complexification may be considered as an alternative to compactification of higher dimensions.

\section{Real Structure of the KN Geometry}

KN metric is represented in the Kerr-Schild (KS) form [15] as follows:

$$
g_{\mu \nu}=\eta_{\mu \nu}+2 h e_{\mu}^{3} e_{\nu}^{3}
$$

where $\eta_{\mu \nu}$ is auxiliary Minkowski background in Cartesian coordinates $\mathrm{x}=x^{\mu}=(t, x, y, z)$,

$$
h=P^{2} \frac{m r-e^{2} / 2}{r^{2}+a^{2} \cos ^{2} \theta}, \quad P=\frac{(1+Y \bar{Y})}{\sqrt{2}},
$$

and $e^{3}(\mathrm{x})$ is a tangent direction to a principal null congruence (PNC), which is determined by the form

$$
e_{\mu}^{3} d x^{\mu}=d u+\bar{Y} d \zeta+Y d \bar{\zeta}-Y \bar{Y} d v
$$

via function $Y(\mathrm{x})$, which is obtained from the Kerr theorem $[15,36-42]$. (Here $\zeta=(x+i y) / \sqrt{2}, \bar{\zeta}=(x-i y) / \sqrt{2}, u=(z+$ $t) / \sqrt{2}$, and $v=(z-t) / \sqrt{2}$ are the null Cartesian coordinates, $r, \theta$, and $\phi$ are the Kerr oblate spheroidal coordinates, and $Y(\mathrm{x})=e^{i \phi} \tan (\theta / 2)$ is a projective angular coordinate. The used signature is $(-+++)$.)
The PNC forms a caustic at the Kerr singular ring, $r=$ $\cos \theta=0$. As a result, the KN metric (1) and electromagnetic potential,

$$
A_{\mu}=-P^{-2} \operatorname{Re} \frac{e}{(r+i a \cos \theta)} e_{\mu}^{3},
$$

are aligned with Kerr PNC and concentrate near the Kerr ring, forming a closed string-waveguide for traveling electromagnetic waves [2, 3, 17, 18]. Analysis of the Kerr-Sen solution to low energy string theory [5] showed that similarity of the Kerr ring with a closed strings is not only analogue, but it has really the structure of a fundamental heterotic string [4]. Along with this closed string, the KN geometry contains also a complex open string, [26-29], which appears in the complex representation of Kerr geometry initiated by Newman [31, 32]. This string gives an extradimension $\theta$ to the stringy source $(\theta \in[0, \pi])$, resulting in its extension to a membrane (bubble source $[9,12]$ ). A superstring counterpart of this extension is a transfer from superstring theory to 11dimensional M-theory and M2-brane [35].

Kerr theorem determines the shear free null congruences with tangent direction (3) by means of the solution $Y(\mathrm{x})$ of the equation

$$
F\left(T^{A}\right)=0
$$

where $F\left(T^{A}\right)$ is an arbitrary holomorphic function in the projective twistor space with $\mathrm{CP}^{3}$ coordinates

$$
T^{A}=\left\{Y, \lambda^{1}=\zeta-Y v, \lambda^{2}=u+Y \bar{\zeta}\right\}
$$

Using the Cartesian coordinates $x^{\mu}$, one can rearrange variables and reduce function $F\left(T^{A}\right)$ to the form $F\left(Y, x^{\mu}\right)$, which allows one to get solution of (5) in the form $Y\left(x^{\mu}\right)$.

For the Kerr and KN solutions, the function $F\left(Y, x^{\mu}\right)$ turns out to be quadratic in $Y$ as follows:

$$
F=A\left(x^{\mu}\right) Y^{2}+B\left(x^{\mu}\right) Y+C\left(x^{\mu}\right)
$$

and (5) represents a quadric in the projective twistor space $\mathrm{CP}^{3}$, with a nondegenerate determinant $\Delta=\left(B^{2}-4 A C\right)^{1 / 2}$ which determines the complex radial distance $[39,43]$ as follows:

$$
\widetilde{r}=-\Delta=-\left(B^{2}-4 A C\right)^{1 / 2} \text {. }
$$

This case is explicitly resolved and yields two solutions

$$
Y^{ \pm}\left(x^{\mu}\right)=\frac{(-B \mp \widetilde{r})}{2 A},
$$

which allows one to restore two PNC by means of (3).

One can easily obtain from (7) and (9) that complex radial distance $\widetilde{r}$ may also be determined from the relation

$$
\widetilde{r}=-\frac{d F}{d Y}
$$


and therefore, the Kerr singular ring, $\tilde{r}=0$, corresponds to caustics of the Kerr congruence as follows:

$$
\frac{d F}{d Y}=0
$$

As a consequence of the Vieta's formulas, the quadratic in $Y$ function (7) may be expressed via the solutions $Y^{ \pm}\left(x^{\mu}\right)$ in the form

$$
F\left(Y, x^{\mu}\right)=A\left(Y-Y^{+}\left(x^{\mu}\right)\right)\left(Y-Y^{-}\left(x^{\mu}\right)\right) .
$$

\section{Complex Kerr Geometry and the Complex Retarded-Time Construction}

KN solution was initially obtained by a "complex trick" [13], and Newman and Lind [31, 32] showed that linearized KN solution may be generated by a complex world line. This complex trick was first described by Appel in 1887 [30] as a complex shift. Appel noticed that Coulomb solution

$$
\phi(\vec{x})=\frac{e}{r}=\frac{e}{\sqrt{x^{2}+y^{2}+z^{2}}}
$$

is invariant under the shift $\vec{x} \rightarrow \vec{x}+\vec{a}$ and considered complex shift of the origin, $\left(x_{0}, y_{0}, z_{0}\right)=(0,0,0)$ along $z$ axis. $\left(x_{0}, y_{0}, z_{0}\right)=(0,0,-i a)$. On the real slice he obtained the complex potential

$$
\phi_{a}(\vec{x})=\operatorname{Re} \frac{e}{\widetilde{r}}
$$

with complex radial coordinate $\tilde{r}=r+i a \cos \theta$. It was shown in $[2,39,43]$ that potential (14) corresponds exactly to $\mathrm{KN}$ electromagnetic field, and the exact $\mathrm{KN}$ solution may be described as a field generated by a complex source propagating along a complex worldline parametrized by complex time $\tau_{L}$ as follows:

$$
x_{L}^{\mu}\left(\tau_{L}\right)=x_{0}^{\mu}(0)+u^{\mu} \tau_{L}+\frac{i a}{2}\left\{k_{L}^{\mu}-k_{R}^{\mu}\right\},
$$

where $u^{\mu}=(1,0,0,1), k_{R}=(1,0,0,-1)$, and $k_{L}=(1,0,0,1)$. Index $L$ labels it as a left structure, and we should add a complex conjugate right structure

$$
x_{R}^{\mu}\left(\tau_{R}\right)=x_{0}^{\mu}(0)+u^{\mu} \tau_{R}-\frac{i a}{2}\left\{k_{L}^{\mu}-k_{R}^{\mu}\right\} .
$$

Therefore, the Kerr geometry may be obtained by the complex shift from the Schwarzschild one, indicating that from the complex point of view the Kerr and Schwarzschild geometries are equivalent and differ only by the relative positions of the real slice, which for the Kerr solution is to be aside of its center. Complex shift turns the Schwarzschild radial directions $\vec{n}=$ $\vec{r} /|r|$ into complex radial directions $\tilde{r}$ of the twisted Kerr congruence (Figure 1).

\section{Complex Kerr's String}

It was obtained [26-29, 33] that the complex world line $x_{0}^{\mu}(\tau)$, parametrized by complex time $\tau=t+i \sigma$, represents

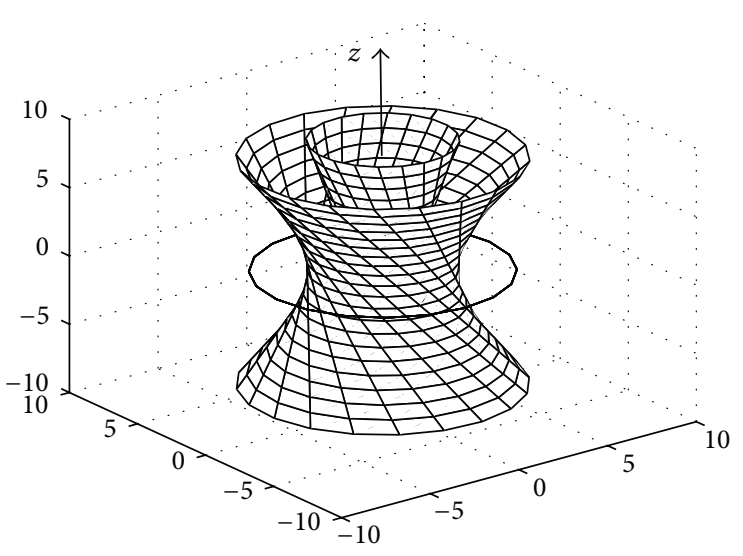

FIGURE 1: Twistor null lines of the Kerr congruence are focused on the Kerr singular ring, forming a two-sheeted spacetime branched by closed string.

really a two-dimensional surface which takes an intermediate position between particle and string. The corresponding "hyperbolic string" equation [33], $\partial_{\tau} \partial_{\bar{\tau}} x_{0}(t, \sigma)=0$, yields the general solution

$$
x_{0}(t, \sigma)=x_{L}(\tau)+x_{R}(\bar{\tau})
$$

as sum of the analytic and antianalytic modes $x_{L}(\tau), x_{R}(\bar{\tau})$, which are not necessarily complex conjugate. For each real point $x^{\mu}$, the parameters $\tau$ and $\bar{\tau}$ should be determined by a complex retarded-time construction. Complex source of the $\mathrm{KN}$ solution corresponds to two straight complex conjugate worldlines, (15), (16). Contrary to the real case, the complex retarded and advanced times $\tau^{\mp}=t \mp \widetilde{r}$ may be determined by two different (left or right) complex null planes (Figure 2), which are generators of the complex light cone. It yields four different roots for the left and right complex structures [39, 43] as follows:

$$
\begin{aligned}
& \tau_{L}^{\mp}=t \mp\left(r_{L}+i a \cos \theta_{L}\right), \\
& \tau_{R}^{\mp}=t \mp\left(r_{R}+i a \cos \theta_{R}\right) .
\end{aligned}
$$

The real slice condition determines correlation between parameter $\sigma=a \cos \theta$ and angular directions $\theta$ of the null rays of the Kerr congruence in the diapason $\theta \in[0, \pi]$. This puts restriction $\sigma \in[-a, a]$ indicating that the complex string is open, and its endpoints $\sigma= \pm a$ may be associated with the Chan-Paton charges of a quark-antiquark pair. In the real slice, the complex endpoints of the string are mapped to the north and south twistor null lines, $\theta=0, \pi$, see Figure 3 .

Orientifold. The complex open string boundary conditions [26-29] require the worldsheet orientifold structure [35, 4447] which turns the open string in a closed but folded one. The worldsheet parity transformation $\Omega: \sigma \rightarrow-\sigma$ reverses orientation of the worldsheet and covers it for the second time in mirror direction. Simultaneously, the left and right modes are exchanged. (Two oriented copies of the interval $\Sigma=[-a, a], \Sigma^{+}=[-a, a]$, and $\Sigma^{-}=[-a, a]$ are joined, 


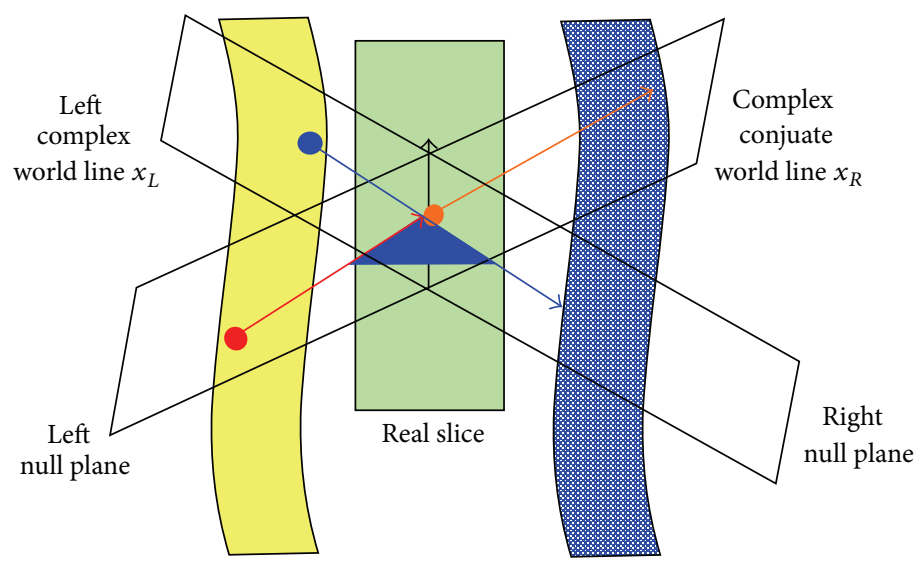

Figure 2: The complex conjugate left and right null planes generate the left and right retarded and advanced roots.

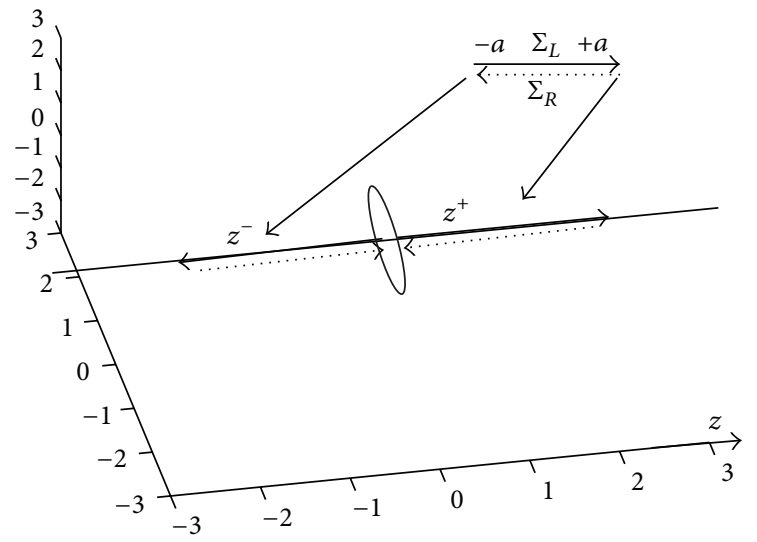

FIGURE 3: Ends of the open complex string, associated with quantum numbers of quark-antiquark pair, are mapped onto the real halfinfinite $z^{+}, z^{-}$axial strings. Dotted lines indicate orientifold projection.

forming a circle $S^{1}=\Sigma^{+} \cup \Sigma^{-}$, parametrized by $\theta$, and map $\theta \rightarrow \sigma=a \cos \theta$ covers the worldsheet twice.) The projection $\Omega$ is combined with space reflection $R: r \rightarrow-r$, resulting in $R \Omega: \tilde{r} \rightarrow-\widetilde{r}$, which relates the retarded and advanced folds of the complex light cones as follows:

$$
R \Omega: \tau^{+} \longrightarrow \tau^{-}
$$

preserving analyticity of the worldsheet. The string modes $x_{L}(\tau), x_{R}(\bar{\tau})$ are extended on the second half cycle by the well-known extrapolation [35, 47] as follows:

$$
x_{L}\left(\tau^{+}\right)=x_{R}\left(\tau^{-}\right) ; \quad x_{R}\left(\tau^{+}\right)=x_{L}\left(\tau^{-}\right),
$$

which forms the folded string, in with the retarded and advanced modes are exchanged every half cycle.

The real KN solution is generated by the straight complex world line (CWL) (15) and by its conjugate right counterpart (16). When the complex string is excited, the orientifold condition (20) becomes inconsistent with the complex conjugation of the string ends, and the world lines $x_{L}(\tau)$ and
$x_{R}(\bar{\tau})$ should be considered as independent complex sources. The projection $\mathscr{T}=R \Omega$ sets parity between the positive Kerr sheet determined by the right retarded time and the negative sheet of the left advanced time. It allows one to escape the antianalytical right complex structure, replacing it by the left advanced one, and the problem is reduced to self-interaction of the retarded and advanced sources determined by the time parameters $\tau^{ \pm}$. For any nontrivial (not straight) CWL, the Kerr theorem will generate different congruences for $\tau^{+}$, and $\tau^{-}$. Each of these sources produces a two-sheeted KerrSchild geometry, and the formal description of the resulting fourfolded congruence should be based on the multiparticle Kerr-Schild solutions [40-42]. (Physical motivation of such a splitting of the sources is discussed in seminal paper by De Witt and Breme [48], where authors introduce the similar "bitensor" fields, which are predecessors of the two-point Green's functions and Feynman propagator. The problem of physical interpretation goes beyond frame of this paper and will be considered elsewhere.) The corresponding twoparticle generating function of the Kerr theorem will be

$$
F_{2}\left(T^{A}\right)=F_{L}\left(T^{A}\right) F_{R}\left(T^{A}\right)
$$

where $F_{L}$ and $F_{R}$ are determined by $x_{L}\left(\tau^{+}\right)$and $x_{L}\left(\tau^{-}\right)$. The both factors are quadratic in $T^{A}$. The corresponding equation

$$
F_{2}\left(T^{A}\right)=0
$$

describes a quartic in $\mathrm{CP}^{3}$ which is the well-known CalabiYau twofold, [35, 47]. We arrive at the result that excitations of the Kerr complex string generate a Calabi-Yau twofold or $\mathrm{K} 3$ surface, on the projective twistor space $\mathrm{CP}^{3}$.

\section{Outlook}

One sees that the Kerr-Schild geometry displays striking parallelism with basic structures of superstring theory. In the recent paper $[17,18]$ we argued that it is not accidental, because gravity is a fundamental part of the superstring theory. However, the Kerr-Schild gravity, being based on twistor theory, displays also some inherent relationships with 
superstring theory, which is confirmed by the principal result of this paper-presence of the Calabi-Yau twofold in the complex twistorial structure of the Kerr geometry.

In many respects the Kerr-Schild gravity resembles the twistor-string theory [24, 49, 50], which is also fourdimensional, based on twistors, and related with experimental particle physics. On the other hand, the complex Kerr string has much in common with the complex critical $N=2$ superstring $[33,47,51]$, which is also related with twistors and has the complex critical dimension two (four real dimensions), indicating that $N=2$ superstring may be consistent with four-dimensional spacetime. However, signature of the $N=2$ string may only be $(2,2)$ or $(4,0)$, which caused the obstacles for embedding of this string in the spacetimes with minkowskian signature. Up to our knowledge, this trouble was not resolved so far, and the initially enormous interest to the $N=2$ string seems to be dampened. Meanwhile, embedding of the $N=2$ string in the complexified Kerr geometry is almost trivial task. It hints that string-like structures of the real and complex Kerr geometry are not simply analogues but reflect the underlying dynamics of the $N=2$ superstring theory.

In the same time, along with wonderful parallelism, the stringy system of the four-dimensional $\mathrm{KN}$ geometry displays very essential peculiarities.

(i) The supplementary Kaluza-Klein space is absent, and the role of compactification circle is played by the Kerr singular ring which realizes a "compactification without compactification" [17, 18].

(ii) The lightlike twistorial rays are tangent to the Kerr singular ring, indicating that the Kerr ring is the lightlike string, playing the role parallel to DLCQ circle in M-theory [52].

(iii) Consistency of the $\mathrm{KN}$ solution with gravitational background of the electron $[8,9,14,17,18]$ shows that the characteristic length of the Kerr ring, $a=$ $\mathrm{J} / \mathrm{m}$, should correspond to the Compton scale of elementary particles. The small masses and great spins of the elementary particles indicate unambiguously that the black hole horizons disappear and the Kerr ring is to be naked. This offers new applications, next to the traditional attention of superstring theory to quantum black holes.

The considered stringy structures of the real and complex Kerr geometry set a parallelism between the 4D Kerr geometry and superstring theory, indicating the potential role of this alliance in the particle physics. On the other hand, these relationships show that complexification of the Kerr geometry may serve an alternative to traditional compactification of higher dimensions in superstring theory.

\section{Acknowledgments}

The author thanks Theo M. Nieuwenhuizen for permanent interest to this work and useful conversations and also very thankful to Dirk Bouwmeester for invitation to Leiden University, where this work was finally crystallized in the process of the numerous discussions with him and members of his group: J. W. Dalhuisen, V. A. L. Thompson, and J. M. S. Swearngin. Author is also very thankful to D. Gal'tsov and L. Järv for discussion at the Tallinn conference "3Quatum" and for the given reference to the related paper [34].

\section{References}

[1] R. P. Kerr, "Gravitational field of a spinning mass as an example of algebraically special metrics," Physical Review Letters, vol. 11, pp. 237-238, 1963.

[2] A. Burinskii, "Microgeons with spin," Journal of Experimental and Theoretical Physics, vol. 39, no. 2, p. 193, 1974.

[3] D. Ivanenko and A. Burinskii, "Gravitational strings in the models of spinning elementary particles," Izv. VUZ. Fiz., vol. 5, p. $135,1975$.

[4] A. Burinskii, "Some properties of the Kerr solution to low energy string theory," Physical Review D, vol. 52, no. 10, pp. 5826-5831, 1995.

[5] A. Sen, "Rotating charged black hole solution in heterotic string theory," Physical Review Letters, vol. 69, no. 7, pp. 1006-1009, 1992.

[6] A. Burinskii, "Instability of black hole horizon with respect to electromagnetic excitations," General Relativity and Gravitation, vol. 41, no. 10, pp. 2281-2286, 2009.

[7] H. Keres, "Physical Interpretation of Solutions of the Einstein Equations," Journal of Experimental and Theoretical Physics, vol. 25, no. 3, p. 504, 1967 (Russian).

[8] W. Israel, "Source of the Kerr metric," Physical Review D, vol. 2, pp. 641-646, 1970.

[9] C. A. L. López, "Extended model of the electron in general relativity," Physical Review D, vol. 30, no. 3, pp. 313-316, 1984.

[10] A. Burinskii, "Supersymmetric superconducting bag as the core of a Kerr spinning particle," Gravitation \& Cosmology, vol. 8, no. 4, pp. 261-271, 2002.

[11] A. Burinskii, "Renormalization by gravity and the Kerr spinning particle," Journal of Physics A, vol. 39, no. 21, p. 6209, 2006.

[12] A. Burinskii, "Regularized Kerr-Newman solution as a gravitating soliton," Journal of Physics A, vol. 43, no. 39, Article ID 392001, 2010.

[13] E. T. Newman, E. Couch, K. Chinnapared, A. Exton, A. Prakash, and R. Torrence, "Metric of a rotating, charged mass," Journal of Mathematical Physics, vol. 6, pp. 918-9920, 1965.

[14] B. Carter, "Global structure of the Kerr family ofgravitational fields," Physical Review Letters, vol. 174, p. 1559, 1968.

[15] G. C. Debney, R. P. Kerr, and A. Schild, "Solutions of the Einstein and Einstein-Maxwell equations," Journal of Mathematical Physics, vol. 10, pp. 1842-1854, 1969.

[16] M. Th. Nieuwenhuizen, "The electron and neutrino as solitons in classical electromagnetism," in Beyond the Quantum, M. Th. Nieuwenhuizen et al., Ed., pp. 332-342, World Scientific, Singapore, 2007.

[17] A. Burinskii, "Gravitational strings beyond quantum theory: electron as a closed heterotic string," Journal of Physics, vol. 361.

[18] A. Burinskii, "Gravity vs. Quantum theory: is electron really pointlike?" Journal of Physics, vol. 343, 2012.

[19] A. Burinskii, "The Dirac-Kerr-Newman electron," Gravitation and Cosmology, vol. 14, no. 2, pp. 109-122, 2008.

[20] A. Burinskii, "Axial stringy system of the Kerr spinning particle," Gravitation \& Cosmology, vol. 10, no. 1-2, pp. 50-60, 2004. 
[21] A. Burinskii, "Kerr geometry beyond the quantum theory," in Proceedings of the Conference 'Beyond the Quantum', M. Th. Nieuwenhuizen et al., Ed., pp. 319-321, World Scientific, Singapore, 2007.

[22] A. Burinskii, "Twistor-beam excitations of Black-Holes and prequantum Kerr-Schild geometry," Theoretical and Mathematical Physics, vol. 163, no. 3, pp. 782-787, 2010.

[23] A. Burinskii, "Fluctuating twistor-beam solutions and holographic pre-quantum Kerr-Schild geometry," Journal of Physics, vol. 222, no. 1, Article ID 012044, 2010.

[24] A. Burinskii, "Twistorial analyticity and three stringy systems of the Kerr spinning particle," Physical Review D, vol. 70, no. 8, Article ID 086006, 2004.

[25] I. Dymnikova, "Spinning superconducting electrovacuum soliton," Physics Letters B, vol. 639, no. 3-4, pp. 368-372, 2006.

[26] A. Burinskii, "String-like structures in complex Kerr geometry," in Relativity Today, R. P. Kerr and Z. Perjés, Eds., p. 149, Akadémiai Kiadó, Budapest, Hungary, 1994, http://arxiv.org/ abs/gr-qc/9303003.

[27] A. Burinskii, Complex String as Source of Kerr Geometry Problems of Modern Physics, Especial Space Explorations vol. 9, 1995, http://arxiv.org/abs/hep-th/9503094.

[28] A. Burinskii, "The Kerr geometry, complex world lines and hyperbolic strings," Physics Letters A, vol. 185, no. 5-6, pp. 441445, 1994.

[29] A. Burinskii, "Kerr spinning particle, strings, and superparticle models," Physical Review D, vol. 57, no. 4, pp. 2392-2396, 1998.

[30] E. T. Whittaker and G. N. Watson, A Course of Modern Analysis, Cambridge University Press, New York, USA, 1996.

[31] E. T. Newman, "Maxwell's equations and complex Minkowski space," Journal of Mathematical Physics, vol. 14, pp. 102-103, 1973.

[32] R. W. Lind and E. T. Newman, "Complexification of the algebraically special gravitational fields," Journal of Mathematical Physics, vol. 15, pp. 1103-1112, 1974.

[33] H. Ooguri and C. Vafa, " $N=2$ heterotic strings," Nuclear Physics B, vol. 361, no. 1, p. 469, 1991, Erratum: Nuclear Physics $B$, vol. 83, pp. 83-104, 1991.

[34] L. M. Dyson, L. Järv, and C. V. Johnson, "Oblate, toroidal, and other shapes for the enhancon," Journal of High Energy Physics, vol. 2002, article 19, 2002.

[35] K. Becker, M. Becker, and J. H. Schwarz, String Theory and MTheory-A Modern Introduction, Cambridge University Press, 2007.

[36] D. Kramer, H. Stephani, E. Herlt, and M. MacCallum, Exact Solutions of Einstein's Field Equations, Cambridge University Press, 1980.

[37] R. Penrose, “Twistor algebra," Journal of Mathematical Physics, vol. 8, pp. 345-366, 1967.

[38] R. Penrose and W. Rindler, Spinors and Space-Time. Vol. 2, Cambridge University Press, Cambridge, UK, 1986.

[39] A. Burinskii and R. P. Kerr, "Nonstationary Kerr congruences," http://arxiv.org/abs/gr-qc/9501012.

[40] A. Burinskii, "Wonderful consequences of the Kerr theorem: multiparticle Kerr-Schild solutions," Gravitation \& Cosmology, vol. 11, no. 4, pp. 301-304, 2005.

[41] A. Burinskii, "The Kerr theorem, Kerr-Schild formalism and multi-particle Kerr-Schild solutions," Gravitation and Cosmology, vol. 12, no. 2, pp. 119-125, 2006.
[42] A. Burinskii, "The Kerr theorem and multi-particle Kerrschild solutions," International Journal of Geometric Methods in Modern Physics, vol. 4, no. 3, Article ID „, p. 437, 2007.

[43] A. Burinskii, "Complex Kerr geometry and nonstationary Kerr solutions," Physical Review D, vol. 67, no. 12, Article ID 124024, p. 11, 2003.

[44] L. Dixon, J. A. Harvey, C. Vafa, and E. Witten, "Strings on orbifolds," Nuclear Physics B, vol. 261, pp. 678-686, 1985, Erratum: Nuclear Physics B, vol. 274, p. 258, 1986.

[45] S. Hamidi and C. Vafa, "Interactions on orbifolds," Nuclear Physics B, vol. 279, no. 3-4, pp. 465-513, 1987.

[46] P. Hořava, "Strings on world-sheet orbifolds," Nuclear Physics B, vol. 327, no. 2, pp. 461-484, 1989.

[47] M. B. Green, J. Schwarz, and E. Witten, Superstring Theory, vol. I, Cambridge University Press, 1987.

[48] B. S. De Witt and R. W. Breme, "Radiation damping in a gravitational field," Annals of Physics, vol. 9, no. 2, pp. 220-259, 1960.

[49] V. P. Nair, "A current algebra for some gauge theory amplitudes," Physics Letters B, vol. 214, no. 2, pp. 215-218, 1988.

[50] E. Witten, "Perturbative gauge theory as a string theory in twistor space," Communications in Mathematical Physics, vol. 252, no. 1-3, pp. 189-258, 2004.

[51] A. D'Adda and F. Lizzi, "Space dimensions from supersymmetry for the $N=2$ spinning string: a four-dimensional model," Physics Letters B, vol. 191, no. 1-2, pp. 85-90, 1987.

[52] L. Susskind, "Another Conjecture about M(atrix) Theory," http://arxiv.org/abs/hep-th/9704080. 

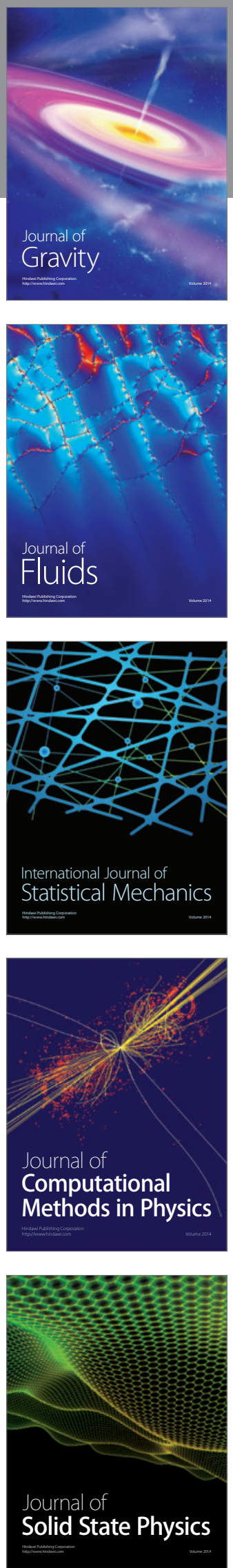

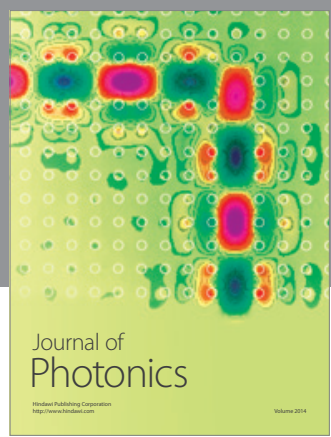

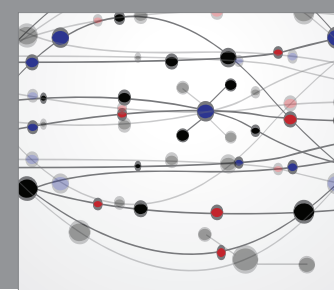

The Scientific World Journal

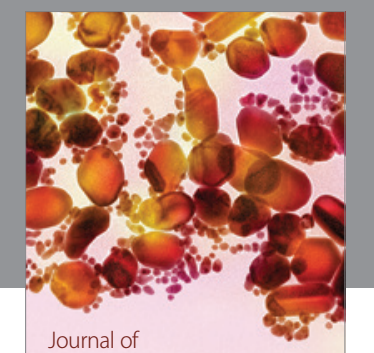

Soft Matter
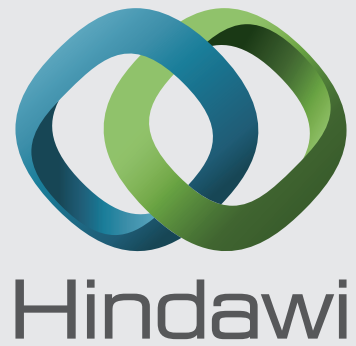

Submit your manuscripts at

http://www.hindawi.com
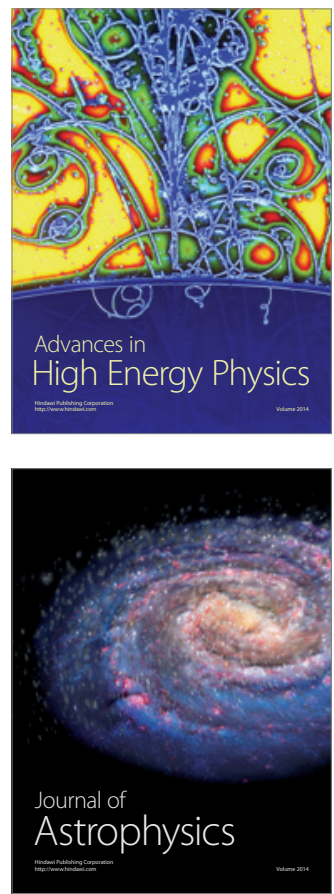
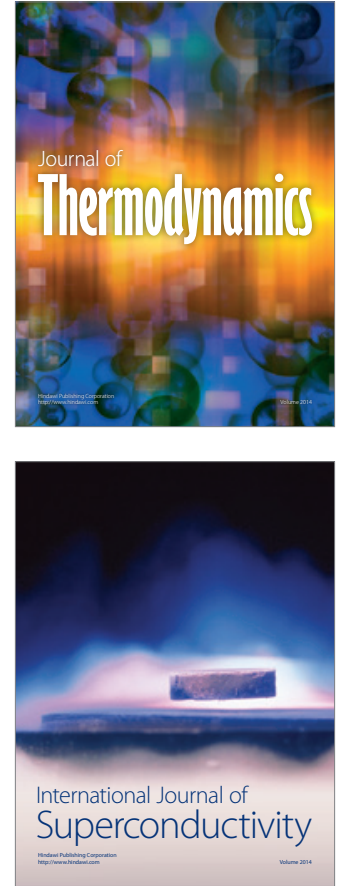
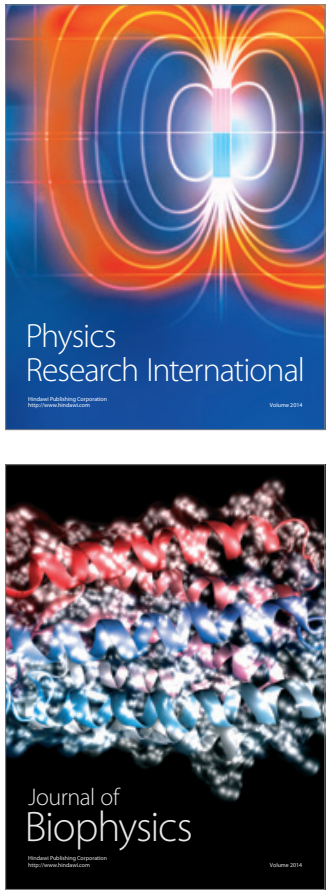
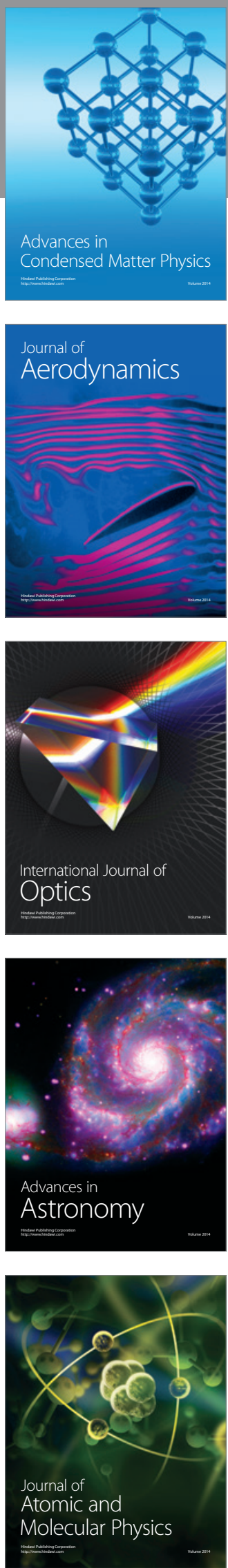